\title{
Éléments rétrospectifs et perspectives pour la didactique des sciences et la didactique du curriculum
}

Retrospective elements and prospects for science education and for the teaching curriculum

\section{Maryline Coquidé}

\section{OpenEdition \\ Journals}

Édition électronique

URL : http://journals.openedition.org/educationdidactique/2550

DOI : 10.4000/educationdidactique.2550

ISSN : 2111-4838

\section{Éditeur}

Presses universitaires de Rennes

\section{Édition imprimée}

Date de publication : 19 décembre 2016

Pagination : 21-31

ISBN : 978-2-7535-5372-9

ISSN : 1956-3485

\section{Référence électronique}

Maryline Coquidé, «Éléments rétrospectifs et perspectives pour la didactique des sciences et la didactique du curriculum », Éducation et didactique [En ligne], 10-3 | 2016, mis en ligne le 19 décembre 2018, consulté le 01 mai 2019. URL : http://journals.openedition.org/educationdidactique/2550 ; DOI : 10.4000/educationdidactique. 2550 


\title{
ÉLÉMENTS RÉTROSPECTIFS ET PERSPECTIVES POUR LA DIDACTIQUE DES SCIENCES ET LA DIDACTIQUE DU CURRICULUM
}

\author{
Maryline Coquidé \\ STEF ENS Cachan et IFE ENS Lyon
}

\begin{abstract}
En m'appuyant sur des travaux de recherche, individuels ou collectifs, j'examine des relations entre didactique (des sciences et du curriculum) et d'autres domaines des Sciences de l'Homme et de la Société (psychologie, sociologie, linguistique et histoire). Je constate néanmoins que les interactions entre didactique et certaines SHS (en particulier anthropologie, phénoménologie et éducation comparée) sont encore insuffisantes. Je discute les exigences ou les modalités de mise en œuvre de ces relations au sein des SHS : des recherches partenariales et un dialogue fondé à la fois sur une complémentarité et une confrontation.
\end{abstract}

Mots-clés : recherche, didactique, sciences, curriculum, sociologie.

Retrospective elements and prospects for science education and for the teaching curriculum

Drawing on individual or collective researches, I examine relationships between science education, science teaching, researches on curriculum and other areas of Human and Society Sciences (psychology, sociology, linguistics and history). I found that interactions between science education and some HSS (especially anthropology, phenomenology and comparative education) are still insufficient. I discuss the requirements or implementation modalities of these relations: research partnerships and dialogue based on both complementarity and confrontation.

Keywords: research, science education, sciences, curriculum, sociology. 


\section{INTRODUCTION}

Ce texte présente un point de vue issu des champs de mes recherches, la didactique des sciences et la didactique du curriculum, qui contribuent aux sciences de l'éducation, elles-mêmes parties prenantes des Sciences de l'Homme et de la Société. Après quelques éléments rétrospectifs situant diverses influences des SHS à l'origine de la didactique des sciences, je présente, en m'appuyant sur quelques travaux de recherche, un développement progressif d'autres regards, portés à l'enseignant et au curriculum en particulier. J'analyse ensuite d'autres dialogues qu'il serait intéressant d'impulser avec les SHS.

\section{QUELQUES ÉLÉMENTS RÉTROSPECTIFS}

\section{Aux origines de la didactique des sciences}

Dans les années soixante-dix en France, la didactique des sciences s'est développée dans un contexte de transformation du système d'éducation et dans un espace inoccupé par des disciplines universitaires déjà constituées, telles la psychologie ou les sciences de l'éducation de l'époque. À son origine, la didactique des sciences a été étroitement liée à l'épistémologie des disciplines et à la psychologie du développement cognitif, avec des fondements constructivistes.

\section{Triple constructivisme et métissage théorique, quelques emprunts}

Les développements de la didactique des sciences ont été contemporains de mouvements constructivistes, un triple constructivisme même, argumente Astolfi (2008). L'idée en a été hétérogène, se nourrissant de trois sources théoriques principales: Piaget, Bachelard et Vygotski. Ce métissage théorique varie selon les cadres de problématisation ou les travaux développés (nous trouvons dès l'origine une didactique plurielle des sciences). Il conduit à se référer à des projets culturels différents : une cognition (Piaget), une conceptualisation (Bachelard) ou une appropriation (Vygotski).

\section{Constructivisme issue de la psychologie cognitive de Piaget}

Dans les débuts des recherches en didactique des sciences, la figure de Piaget dominait. Les études empiriques piagétiennes sur les conceptions des enfants ont, en effet, nourri les recherches didactiques des sciences émergentes. Piaget recherchait quels sont les « schèmes » de pensée que construisent l'enfant et l'adolescent, et par quels « stades » réguliers s'effectue la genèse du développement intellectuel. Le développement n'est pas pour lui l'apprentissage, et l'atteinte d'un certain niveau de développement est la condition nécessaire de certains apprentissages. Le projet Piagétien porte sur le sujet épistémique, c'està-dire le noyau cognitif commun à tous les individus quelle que soit leur histoire individuelle, et il vise une cognition. Il concerne les "invariants opératoires », c'est-à-dire les opérations mentales dont il postule qu'elles sont en jeu à un stade donné, quelle que soit l'activité. Il soumet aux jeunes sujets des problèmes très variés (bûchettes, pâte à modeler, plans inclinés, transvasement de liquides, flottaison d'objets...) mais il s'intéresse moins aux concepts correspondants qu'au développement sous-jacent de l'abstraction. Le terme cognitif insiste sur la nature des opérations logiques, considérées indépendamment des contenus et transversales par rapport à eux. Sa longue accumulation de données empiriques sur les représentations de très nombreux phénomènes a pu être exploitée par les didacticiens qui en ont renversé la perspective, pour la mettre au service du problème d'apprentissages conceptuels spécifiques.

Par ailleurs, chez Piaget, la construction mentale apparaît progressive : le passage de stades suppose discontinuités, déséquilibres et réagencements, mais l'ensemble prend bien l'allure d'une progression vers la maîtrise des outils de la logique formelle et du raisonnement hypothético-déductif. Piaget accorde une grande importance au « dialogue avec les objets », aux expériences fortuites que tout enfant effectue aux prises avec la réalité du monde qui l'entoure, et une place limitée aux interactions sociales. Le didacticien des sciences se positionne plutôt dans l'organisation des rencontres scolaires des objets et des phénomènes et il privilégie les interactions sociales. 


\section{Constructivisme issue de l'épistémologie de Bachelard}

Sans nier l'apport de Piaget, les travaux de didactique des sciences ont développé une perspective épistémologique constructiviste (en particulier Bachelard et Canguilhem). Là non plus, l'emprunt n'est pas direct puisque cette approche a pris naissance dans la philosophie des sciences et l'épistémologie, pour donner du sens aux lenteurs et ratés de la construction historique des concepts scientifiques.

Une des idées-clés reprises de Bachelard est celle d'obstacle (non une difficulté, mais une facilité de l'esprit qui se précipite vers une explication toute prête). Bachelard attire l'attention sur la nécessité d'une rupture avec le sens commun pour pouvoir apprendre, d'un renoncement à ce qu'on croit déjà savoir. Le projet Bachelardien s'intéresse aussi à un sujet épistémique. Il vise une conceptualisation, avec la construction du savoir dans chaque domaine scientifique précis (des connaissances régionales et non générales). Il accorde une grande importance à la construction de problèmes scientifiques et à la démarche de problématisation.

\section{Constructivisme issu de la psychologie sociale de Vygotski}

L'emprunt à Vygotski en didactique des sciences a été plus récent (années quatre-vingt-dix), la redécouverte de l'œuvre de ce psychologue russe ne remontant qu'aux années quatre-vingt, après un demi-siècle d'éclipse. Le marxisme qui l'anime le sensibilise à l'importance des interactions humaines et sociales dans l'apprentissage.

Vygotski place l'apprentissage en position pionnière d'un développement qui en est le fruit. La mission de l'école est alors de stimuler les potentialités du sujet, de le « tirer en avant» du point de vue cognitif. Pour lui, le mouvement fondamental de la pensée va du collectif vers l'individuel. Chaque conquête intellectuelle survient deux fois dans l'histoire du sujet : d'abord propriété collective du groupe, elle s'intériorise grâce à la maîtrise du langage en devenant une conquête individuelle. Pour comprendre le développement du jeune enfant, dans la perspective ouverte par Vygotski, il ne faut l'extraire ni des contextes socio-historiques, qui mettent à sa disposition les outils culturels, ni des contextes inter-subjectifs, à l'intérieur desquels, avec l'aide d'autrui, il apprend à les mettre en œuvre.

\section{Trois constructivismes et trois types d'obstacles}

En combinant ces trois registres constructivistes, les recherches de didactique des sciences ont développé des problématisations variées. Elles ont exploré de différentes façons les enjeux et les défis d'un enseignement triplement constructiviste, en reprenant les termes d'Astolfi (2008), avec des problématiques articulant des orientations à la fois épistémologique, psychologique et pédagogique :

- un constructivisme épistémologique : avec un appui important sur Bachelard et sur Canguilhem lorsqu'on a cherché, en premier lieu, à caractériser des obstacles épistémologiques qu'il faut dépasser pour maîtriser les concepts spécifiques d'un champ scientifique donné. L'histoire des sciences a fourni des éclairages utiles pour montrer à quoi on s'affronte dans ce domaine du savoir ;

- un constructivisme psychologique : avec un emprunt à Piaget lorsqu'on a voulu déterminer, dans une optique développementale, les possibilités cognitives d'un apprentissage chez de jeunes élèves. A dominé ici l'idée d'obstacles psychogénétiques, témoins de l'inachèvement provisoire de la pensée abstraite ; - un constructivisme pédagogique : avec une référence majeure à Vygotski, dès lors qu'était en jeu une dimension collective des apprentissages, la conception de dispositifs didactiques, ou bien l'analyse d'obstacles didactiques créés par des traditions de l'enseignement.

\section{Pour débattre : peu de perspectives sociales}

Aux origines, la didactique des sciences avait peu développé de perspectives sociales. S'intéressant à la construction de programmes d'enseignement en sciences et en technologie, Martinand (1986) avait cependant montré une limite du constructivisme : son incapacité à spécifier des contenus adaptés aux contextes socio-économiques. Le concept de pratique sociale de référence a permis d'attirer l'attention sur le fait que, à côté des contraintes d'ordre psychogénétique et épistémologique, l'enseignement a à prendre en compte des enjeux sociaux. Par exemple, quelle image y donne-t-on du travail et des pratiques scien- 
tifiques d'aujourd'hui ? Pour Martinand, la notion de "situation » cachait le fait que les engagements des acteurs étaient extrêmement variés. Il préférait le terme de " mode d'activité didactique » pour désigner le pôle organisateur de l'activité des élèves par l'enseignant, ce qui permettait de ne pas exclure l'interaction entre ce qui est proposé à l'élève et la façon dont celui-ci s'en empare. Analyser l'éducation comme un fait social conduit, en outre, à considérer l'ensemble de la scolarité comme la transmission générationnelle d'une culture. Cette transmission culturelle et sociale ne peut pas être la somme des petites transmissions cognitives individuelles. Elle conduit à envisager une approche curriculaire, et pas seulement disciplinaire, des contextes scolaires et non scolaires.

\section{LE DÉVELOPPEMENT D'AUTRES « REGARDS »}

Par la suite, des travaux de didactique des sciences et des techniques ont développé d'autres " regards ", avec des problématisations centrées sur des acteurs (élèves ou enseignants, et pas seulement en tant que sujet épistémique), sur des interactions et sur le groupe (perspectives psycho-sociales et historico-culturelles), sur les organisations et les institutions (perspectives plus sociologiques), assortis de leurs systèmes de références propres.

L'approche microsociologique du rapport au savoir a, par exemple, joué un rôle heuristique dans plusieurs recherches en didactique des sciences (Lebeaume \& Coquidé, 2002). Avec cette notion, l'élève n'était plus perçu dans une dimension essentiellement épistémique mais comme un sujet évolutif, inscrit dans un environnement complexe, avec un prise en compte de dimensions sociétales (sociales, culturelles, familiales, institutionnelles...) et psychologiques (cognitives, psychanalytiques), en particulier pour mieux comprendre des différenciations d'apprentissage.

Les problématisations didactiques ont pu ainsi intégrer d'autres registres: notamment celui des dimensions éthiques ou affectives, avec une ouverture plus importante aux questions relatives à l'acte d'enseignement et à la sémiotisation,

Ce séminaire sur le dialogue avec les Sciences Humaines et de la Société est donc une occasion de débattre de certains points : les analyses langagières des activités scolaires, la question de la professionnalité de l'enseignant, la perspective curriculaire et historico-culturelle.

\section{Les valeurs ou des dimensions affectives}

Prenons deux exemples de thèses en didactique traitant du rapport au vivant : celle de Michèle dell'Angelo (2007) et celle de Michel Vidal (2014). Ces deux cas permettent d'illustrer une mobilisation, dans des recherches didactiques, d'aspects éthiques ou politiques, avec des emprunts théoriques en Sciences de l'Homme et de la Société, sociologie et philosophie en particulier.

Dans sa thèse, Michèle dell'Angelo (2007) a étudié l'appropriation, en milieu scolaire, d'un rapport scientifique et éthique au vivant, et aussi d'autres rapports au vivant construits en milieu familial et social. Un suivi longitudinal d'une cohorte d'élèves du CM2 à la $6^{\mathrm{e}}$ de plusieurs classes a permis d'évaluer l'effet de l'enseignement de SVT de différents établissements. La méthode d'analyse a été fondée sur l'identification de registres mobilisés par les élèves face à des vivants (registre affectif, pratique, cognitif, esthétique, moraliste...).

C'est à l'éducation au bien-être animal dans l'enseignement agricole et au développement d'une empathie interspécifique que s'est intéressé Michel Vidal (2014). Le choix du secteur d'enseignement agricole était particulièrement pertinent : des élèves souvent d'origine rurale, aux motivations professionnelles variées et divers vécus familiaux et sociaux concernant les animaux, une diversité des secteurs de formation qui permet d'étudier le problème pour l'animal de production ou pour l'animal de compagnie, une formation professionnelle de type alternance (des enseignements de zootechnie et des stages qui permettent d'expériencer différentes relations et pratiques). Ce travail a mobilisé deux concepts polémiques : le bien-être animal, qui relève d'une question socialement vive, avec différentes éthiques et représentations de l'homme et de l'animal, et l'empathie, concept polymorphe et polysémique, lui aussi sujet à des divergences de conceptions et porteur de controverses. Afin d'identifier des facteurs pouvant influencer l'apprentissage de l'empathie dans le système éducatif, Michel Vidal a proposé une modélisation d'empathie interspécifique, fondée sur une relation entre sujets (sujet humain et sujet animal) multi-référentielle (sensorielle, cognitive, affective, éthique). 


\section{Les dimensions socio-historiques et historico-culturelles}

Bernard Schneuwly (2008) rappelle que l'élaboration vygotskienne permet de prendre en compte à la fois une dimension socio-constructiviste (avec l'importance accordée aux interactions langagières) et une dimension historico-culturelle (avec la construction d'outils culturels). Cette perspective vygotskienne de travaux didactiques conduit à mobiliser des méthodologies d'analyses langagières. Ce filtre du travail langagier permet de mettre en évidence une dimension d'interpsychologie dans le débat en classe et peut aussi ouvrir une question plus sociologique des malentendus scolaires générateurs d'inégalités d'apprentissage (Bautier \& Rayou, 2010).

\section{Les analyses langagières des activités scolaires}

Le langage représente une matière première importante du champ des SHS (traduit par un corpus de comportements, de discours, de témoignages...). Les analyses langagières de situation de classe se sont développées selon différents cadres théoriques linguistiques (par exemple cadre interactionniste de Kerbrat-Orecchioni ou cadre dialogique de Bakhtine). Elles rencontrent aussi des limites : les pratiques scientifiques sont, par exemple, définies à la fois par les pratiques matérielles du domaine, par le versant social de son élaboration et par les pratiques discursives dans lesquelles celles-ci prennent forme. Tout rapport scientifique au monde est doublement médiatisé : par l'outil et par le langage. Les pratiques langagières d'une communauté scientifique sont certes importantes mais ne représentent qu'une facette des activités scientifiques. Au niveau scolaire, certaines activités de sciences, tels les travaux pratiques, sont difficilement traduisibles uniquement avec des transcripts.

\section{Professionnalité et spécialité de l'enseignant}

À la suite de la mise en place des IUFM (1989), les travaux sur les enseignants et sur la formation ont pris de l'ampleur avec, en particulier, une mobilisation de l'ergonomie, de la psychologie du travail, de la sociologie ; le développement de recherches sur la professionnalité, et l'élaboration d'une didactique professionnelle. Comment les recherches de didactique des sciences sur l'enseignant se situent-elles dans ce contexte?

Prenons, par exemple, l'ouvrage d'Isabelle Vinatier (2009) qui vise à présenter une didactique professionnelle de l'enseignement. Il articule trois cadres théoriques : théorie de l'activité (Vergnaud), linguistique (Kerbrat-Orecchioni) et didactique professionnelle (Pastré), pour élaborer une définition de l'identité enseignante, construite sur la personne mais surtout sur l'activité en situation (identité-enacte). L'analyse de l'activité enseignante est construite à partir d'analyse de pratiques (corpus d'entretiens en ateliers d'analyse de pratiques d'IUFM). Ces supports privilégiés de recherche ont des incidences : corpus sur l'enseignement primaire, centration sur le pédagogique et sur certaines matières phares du primaire (les sciences sont peu présentes, absence de l'EPS et des pratiques artistiques). Dans cette perspective de didactique professionnelle, enseigner est pris au sens intransitif. L'activité enseignante y est appréhendée en acte mais assez indépendante des contenus d'enseignement. Une place centrale, dans l'analyse de ces pratiques, est accordée au langage ; en particulier aux moments d'interactions en présence enseignant-élève et à l'activité dialogique. En conséquence, un accent est donné ici aux régulations de type pédagogique et à l'accompagnement de l'élève dans des moments particuliers (aide aux élèves en difficulté, soutien scolaire...).

Dans une perspective didactique, enseigner est transitif (enseigner... quelque chose, un enseignement de...). Nous pouvons souligner aussi l'intérêt de ne pas occulter le temps curriculaire (les finalités d'un enseignement ne peuvent apparaître que sur une temporalité plus longue). Il apparait alors une nécessité de problématiser des recherches didactiques sur les enseignants en termes de spécialité (Lebeaume, 2007) et pas seulement de professionnalité. Pour Joël Lebeaume (2007), la discipline de spécialité des enseignants constitue une matrice des actions professionnelles en relation avec une diversité des contenus et leurs spécificités, à la fois en tant que façon de penser et de manière d'agir. Les spécialités professionnelles, signalées par les désignations «professeur de...», spécialiste d'un segment scolaire (professeur des écoles) ou spécialiste d'un enseignement disciplinaire (pour le second degré), façonnent les pratiques des enseignants. Des spécificités sociales, épistémologiques et professionnelles 
précisent ces spécialités enseignantes. Ces spécialités sont également marquées par l'organisation sociale des personnels enseignants en corps professoraux et en associations de spécialistes. Signalons aussi les travaux de Joël Bisault (2011) concernant la spécialité d'un enseignant du primaire pour enseigner les sciences et évoquons les travaux relatifs à l'épistémologie pratique du professeur portés par la Théorie de l'Action Conjointe Didactique de Gérard Sensevy (2011) (par exemple : Marlot \& Toullec-Théry, 2014 ; Marlot \& Morge, 2015).

\section{Une perspective curriculaire}

La didactique des sciences s'intéresse à l'éducation et à la diffusion, d'un point de vue d'observateur externe qui veut décrire et comprendre, et aussi d'un point de vue d'intervenant qui veut construire des dispositifs scolaires (programmes d'enseignement, dispositifs d'évaluation, curriculum...) ou non scolaires (pour une culture scientifique, les projets éducatifs et culturels, les expositions scientifiques...). Des recherches didactiques conduisent à impulser une ouverture spatiale (pas seulement la classe) et temporelle (pas seulement la séquence de classe) des analyses, avec des travaux à temporalités plus amples, qu'elles soient individuelle (biographies), organisationnelle ou historique. Citons, par exemple, l'étude des récits de vie d'enseignants de biologie au Québec et en France (Bernard, 2008) ou l'analyse conjointe, didactique et sociologique, de devoirs de SVT (Rayou \& Sensevy, 2015).

À côté de ces questions, les recherches didactiques étudient aussi les constructions, les cohérences et les mises en œuvre de curriculum. La didactique y développe, là aussi, un point de vue centré sur les contenus, même si disciplines et curriculums en France sont souvent pensés comme apprentissage de savoirs et même de savoirs discursifs (Forquin, 2008 ; Martinand, 1986, 2000). Penser curriculum, c'est penser à la fois ce qui va être enseigné, l'organisation, la programmation temporelle, les ressources (humaines, spatiales, matérielles, relationnelles) qui sont mises en œuvre et, finalement, ce qui va être appris et évalué, à court et à plus long terme. C'est aussi envisager des possibilités de reconfigurations curriculaires ou disciplinaires. Prenons deux exemples.

\section{Exemple 1 : l'enseignement scientifique et technologique du collège}

C'est principalement par une enquête historique que Lebeaume $(2008,2011)$ reconstruit l'enseignement scientifique et technologique du collège. La recherche sur l'évolution historique des constructions curriculaires croise ici des analyses à la fois didactiques et sociologiques ou sociopolitiques, pour mettre en évidence des coordinations et des différenciations des enseignements scientifiques et techniques. Cette approche, qui peut être désignée comme historico-didactique, questionne l'organisation des contenus, structurés en disciplines ou non, les évolutions de celle-ci, les progressions cognitives dans chaque discipline scolaire. Elle porte attention aux comparaisons internationales, aux mouvements historiques des configurations disciplinaires et curriculaires, aux mutations contemporaines des contenus, des pratiques et des institutions scientifiques.

\section{Exemple 2 : deux études d'Enseignement Intégré de Science et Technologie (EIST)}

Je propose ici de mettre en regard deux études d'EIST présentant des approches complémentaires, sociologique (Harlé \& Lanéelle, 2011) et didactique (Coquidé, Fortin \& Lasson, 2013).

La problématique de l'étude d'EIST réalisée par Harlé et Lanéelle (2011) a porté sur différentes questions. Quelles sont les formes d'enseignement intégré ? Comment ces expérimentations sont-elles impulsées? Quels sont les contextes facilitant la mise en œuvre de ces projets? Comment les enseignants se mobilisent-ils ? Comment mettent-ils en place un travail collaboratif ? La recherche s'est appuyée sur une enquête qualitative, fondée sur des entretiens semi-directifs avec des chefs d'établissement et des membres des équipes enseignantes engagées dans ces projets ; l'observation de la configuration des relations professionnelles, avec une approche née de l'analyse des réseaux sociaux des membres des équipes lors de réunions; la collecte des « cahiers de l'innovation » auprès des enseignants. L'étude conduit à constater que les disciplines concernées (SVT, Physique-Chimie, Technologie) semblent être à même de constituer un champ curriculaire favorable à l'innovation. Mais cela ne suffit pas. La place 
institutionnelle apparait fondamentale et la présence d'un enseignant-leader apparaît facilitateur, à condition que le réseau socioprofessionnel constitué permette un véritable travail d'équipe. Cette étude se place dans un cadre sociologique : sociologie du curriculum, sociologie des réseaux et sociologie des transformations.

L'étude de Coquidé, Fortin et Lasson (2013) s'est, de son côté, intéressée à la dynamique de construction curriculaire d'EIST et au processus d'élaboration d'enseignement par l'équipe enseignante d'EIST de trois collèges. L'analyse des séances hebdomadaires de concertation, entre les trois enseignants de différentes disciplines de l'équipe EIST, a conduit à repérer un espace participatif d'échanges et de négociations pour l'élaboration d'un curriculum local auto-prescrit. La tentative d'EIST y apparait comme un enseignement en tension qui vise à promouvoir une nouvelle unité d'enseignement, tout en maintenant un pluralisme disciplinaire. Les écarts entre le curriculum auto-prescrit élaboré par l'équipe et les curriculums coproduits au niveau de la mise en œuvre, tout au long de l'année scolaire, dans chaque classe et par chaque enseignement, ont été analysés. Chaque enseignant a mis en œuvre le curriculum auto-prescrit en l'adaptant à sa classe, en fonction du questionnement des élèves et selon son habitus, ce qui a conduit à considérer un effet de spécialisation disciplinaire dans le curriculum coproduit d'EIST de chacun de ces professeurs. Cette étude se place dans un cadre didactique : didactique des sciences et techniques, didactique du curriculum.

En rapprochant ces deux études, émerge la question de la conjugaison, de la complémentarité et la confrontation, des approches didactiques et sociologiques, avec les potentialités d'analyses croisées prenant en compte les dimensions épistémologiques et sociales des curriculums.

\section{Pour débattre}

L'évocation de ces quelques cas témoigne du développement de nouvelles perspectives de travaux didactiques, par exemple historico-didactique ou socio-didactique. Un premier ensemble d'interrogations émerge cependant. Que peut signifier ces adjectifs juxtaposés, avec ou sans tirets, attribués à ces nouvelles perspectives? Une articulation? Une reconfiguration? Une coordination des perspec- tives ? Est-ce au niveau de la problématisation des recherches? De l'analyse du corpus ? Comment exploiter une vraie richesse de l'altérité au sein des SHS, favoriser les échanges sur une question, tout en restant vigilant face à un risque de pulvérisation ou de dilution de l'objet de recherche?

Patrick Rayou (2014), lors du précédent séminaire de l'ARCD, avait discuté les potentialités d'un espace commun (didactique et sociologie) de problématisation. Qui dit espace commun, dit au sein d'une équipe ou entre personnes de culture différente. Comment s'articulent alors les contributions ? Par quel dialogue? Les échanges sont-ils complémentaires ? Par addition ou par confrontation? Comment se transforme ou évolue cet espace commun de problématisation ? La proposition de distinguer professionnalité (didactique professionnelle) et spécialité professionnelle (didactique), la comparaison de différentes études, sociologiques et didactiques, d'EIST peuvent illustrer une fécondité, pour la didactique, de faire avec et contre au sein des SHS, avec une nécessité d'affinement de la problématisation.

\section{D'AUTRES « DIALOGUES » À IMPULSER}

Dans un article fondateur de la didactique comparée (2002), Alain Mercier, Maria SchubauerLeoni et Gérard Sensevy concluaient :

« La didactique, dès lors qu'on la conçoit comme une anthropologie des pratiques d'éducation, est organiquement, nécessairement comparatiste. La didactique comparée devrait pouvoir se rendre capable de tisser des liens inédits avec d'autres champs de recherche: en sciences de l'éducation avec, notamment, l'éducation comparée; à l'intérieur des sciences de l'homme et de la société, avec l'ensemble des disciplines anthropologiques $»\left(\right.$ p. 14) ${ }^{1}$.

Un tissage de liens avec l'éducation comparée et avec les disciplines anthropologiques était préconisé. Quinze ans plus tard, cependant, ces tissages semblent encore ténus ou hors de la perspective didactique, comme en témoignent nos analyses ci-après, concernant l'anthropologie, la phénoménologie et l'éducation comparée. 


\section{Dimension anthropologique}

Il y a des conceptions de l'homme et de la société qui sont en jeu à travers les questions de savoirs, de curriculum et de disciplines, de constructions et d'interactions dans l'espace scolaire. Quelles sont les investigations didactiques qui supposent la prise en compte du petit humain, dans ses expériences, scolaires et disciplinaires, dans ses découvertes et ses apprentissages scientifiques et technologiques? Michel Develay (1997) avait commenté une approche anthropologique des savoirs scolaires :

« Une anthropologie des savoirs scolaires conduirait à regarder quelles questions sont posées aujourd'hui par une discipline déterminée au regard de son projet initial et quelle vision de l'humain est ainsi dévoilée » (p. 65).

Une dimension psycho-anthropologique peut en effet être très constructive, par rapport à la construction du sens pour le sujet, sans oublier la question de la langue, vecteur linguistique et idiome forcément national.

Prenons, par exemple, le cas de l'espace, du temps et des relations spatio-temporelles, constamment utilisés dans tous les champs des sciences et de la technologie (Coquidé \& Prieur, 2010 ; Coquidé \& Morge, 2011). Si « espace » et «temps » sont des concepts scientifiques, appréhendés de façon extrinsèque par différentes techniques de mesure, ce sont aussi des constructions sociohistoriques et culturelles, des perceptions et des sentiments. Une approche d'anthropologie cognitive décrit ainsi la façon dont les différents systèmes linguistiques et culturels influencent la représentation que se font les individus de l'espace et du temps. D'une façon pionnière, l'anthropologue américain Edward Hall, spécialiste de communication interculturelle, a ainsi étudié des "dimensions cachées » de toute civilisation. Il a examiné la façon dont le temps est vécu dans différentes cultures. Il y compare un temps linéaire, unidimensionnel avec une succession d'activités, des civilisations des pays du Nord, et un temps pluridimensionnel des cultures du Sud (Hall, 1983 / 1984). La façon dont Hall reprend le travail de Piaget, en l'envisageant comme un processus d'acculturation occidentale, peut aussi interpeler le didacticien. Pour cet anthropologue, quand Piaget étudie comment les enfants apprennent les règles fondamentales de fonctionnement de temps et d'espace, ils apprennent en même temps notre propre système de logique occidentale. Il considère, par là, la méthodologie Piagétienne comme satisfaisante pour le mode de pensée linéaire occidental, limitée cependant à notre culture (Hall, 1984, p. 168 et suivantes). En outre, les langues que nous parlons modifient notre façon de percevoir le monde et nos capacités cognitives. Loin de désigner des référents du monde réel, elles contribuent, elles-mêmes, à l'origine des catégories d'espace et de temps par une opération de désignation. Les représentations spatiales et temporelles vont donc dépendre aussi de la langue d'enseignement.

\section{Dimension phénoménologique}

Une dimension phénoménologique porte l'attention aux dimensions les plus corporelles et sensuelles de l'action. Refusant une réduction de la pensée à la seule discursivité, une posture phénoménologique considère l'enracinement de l'individu dans la vie et l'omniprésence de son corps, fondement d'un sujet distinct d'un monde objectif. Cette dimension dépasse nettement l'ici et maintenant de l'interaction, en faisant place au corps, aux sens et à l'expérience vécue (qui correspond ici à une connaissance subjective et ressentie dont le caractère unique interdit l'interchangeabilité des sujets). Ce qui mérite attention alors n'est pas seulement ce que l'on fait, mais à quel point on y met du sien et à quel point on est imprégné par l'environnement. Tout cela est vécu et accompli dans et à travers le corps, et non dans un discours rationnel, ni dans une conversation avec les autres.

Commentant les méthodologies ethnographiques, le sociologue américain Jack Katz (1999) prend le cas de l'analyse de vidéo et l'exemple de la petite Rachel faisant des puzzles en maternelle. Il argumente la nécessité d'articuler différentes dimensions, dont une dimension phénoménologique. Pour Katz, chaque instant de la vie sociale comporte trois aspects : l'aspect interactionnel, la praxis, et le sens transcendant du moment qui se déploie de façon cachée. Étudier à la fois l'action située et les projets de l'acteur qui la dépassent est, pour lui, un défi que doit affronter aujourd'hui la recherche.

Sensibilisée aux dimensions anthropologiques et phénoménologiques dans un point de vue didactique, j'étudie actuellement, pour une compréhension renouvelée de l'éducation biologique, l'intérêt 
du sens donné aux activités scolaires, avec aussi une attention aux dimensions corporelles et sensuelles de l'action (Coquidé, 2015, sous presse).

\section{Éducation comparée}

L'éducation comparée est peu mobilisée dans les perspectives didactiques. Selon Élisabeth Régnault (2015), deux approches comparatives caractérisent l'éducation comparée : l'approche pragmatique vise l'universalité des pratiques ainsi que la possibilité de transfert de "bonnes pratiques ", tandis que l'approche compréhensive est centrée sur la compréhension des phénomènes dans leur contexte culturel. C'est cette approche compréhensive qui peut particulièrement interpeller le didacticien. Des travaux analysent en détail, par exemple, les répercussions de la culture éducative nationale sur les apprentissages et le rapport des élèves à l'école (Osborn et al., 2003). Ces recherches, en rappelant que les objectifs cognitifs divergent d'un pays à l'autre, éclairent les résultats des classifications internationales. Elles font aussi ressortir des cultures d'apprentissage distinctes, qui rendent plus ou moins acceptable l'incertitude, la prise de risque ou bien l'échec. Mieux connaitre les contextes culturels des enseignements informe les perspectives didactiques comparatistes.

Conséquence d'une visée d'intégration républicaine, la France semble, pour le moment, rester peu concernée par un contexte multiculturel, par rapport à d'autres pays. Un contexte multiculturel peut cependant avoir des conséquences sur l'enseignement, en particulier des sciences de la vie, avec les enseignements relatifs au racisme, à la connaissance du corps humain ou bien encore à l'évolution du vivant. Tout proche de nous, en Belgique, la notion de laiicité est différente de celle de la France : il n'y a pas de séparation entre l'Église et l'État mais des relations contractuelles entre l'État et diverses religions. Laurence Perbal (2009) y a étudié des difficultés spécifiques pour enseigner l'évolution du vivant. Autre exemple, canadien cette fois-ci : la diversification des communautés en Ontario a conduit le gouvernement provincial à revoir les curriculums, pour que ceux-ci soient plus inclusifs et reflètent mieux la diversité de la population. Mais dans les faits, les curriculums scientifiques prennent peu en compte cette injonction d'attention au multicultura- lisme, y compris la vigilance à une prévention antiraciste (Mujawamariya et al., 2014).

\section{EN GUISE DE CONCLUSION}

« Il est important de transgresser les frontières entre sociologie et didactiques », argumente Philippe Losego (2014). Mais s'agit-il vraiment de « transgression »? Davantage que des limites ou des barrières, les frontières représentent aussi des zones de passage ou d'influence. Pour développer un dialogue entre didactique et sociologie, Patrick Rayou (2014) propose d'impulser un espace commun de problématisation ou une problématisation conjointe. Ce que nous devons alors penser et savoir ménager, c'est le partenariat en recherche. Les recherches partenariales posent, en effet, de redoutables problèmes institutionnels et épistémologiques. Quels sont les problèmes qui exigent tel ou tel découpage de la réalité et donc qui supposent la constitution d'équipes de recherches interdisciplinaires ou co-disciplinaires, avec la contribution spécifique des didacticiens? Pour éviter une dilution incontrôlée dans des problématiques par nature engluées dans la sphère sociale, psychologique et anthropologique, cette élaboration conjointe de problématisation doit se faire, comme je l'ai discuté auparavant, avec et contre les autres SHS, le dialogue représente complémentarité mais aussi confrontation. Le rôle des manifestations scientifiques soutenues par l'ARCD et la place de la revue Éducation et Didactique, pour favoriser cet espace partenarial, sont alors à souligner.

\section{NOTES}

1. C'est moi qui, dans cette citation, souligne « éducation comparée » et « disciplines anthropologiques». 


\section{RÉFÉRENCES}

Astolfi, J.-P. (2008) La saveur des savoirs. Disciplines et plaisir d'apprendre. Paris : ESF.

Bautier, É., \& Rayou, P. (2010). Les inégalités d'apprentissage. Programmes, pratiques et malentendus scolaires. Paris : Presses universitaires de France.

Bernard, M.-C. (2008). Les approches du vivant à travers les récits de vie d'enseignants et enseignantes de biologie au collège et au lycée. Thèse université Laval Québec et université Paris 5.

Bisault, J. (2011). Contribution à l'élaboration curriculaire d'une éducation scientifique à l'école primaire : modélisation des moments scolaires à visée scientifique. Mémoire d'HDR, ENS de Cachan.

Osborn, M., Broadfoot, P., McNess, E., Planel, C., Ravn, B., \& Triggs, P. (2003). A World of Difference? Comparing learners across Europe. Maidenhead: Open University Press.

Coquidé, M., \& Prieur, M. (dir.) (2010). Enseigner l'espace et le temps à l'école et au collège. Obstacles, pratiques, outils. Lyon : INRP, Collection Didactiques, Apprentissages, Enseignements.

Coquidé, M., \& Morge, L. (2011). Introduction. Espace et temps dans l'enseignement des sciences et des technologies. Recherches en Didactique des sciences et des technologies, 4, 9-28.

Coquidé, M., Fortin, C., \& Lasson, C. (2013). D'un curriculum auto-prescrit à des curriculums co-produits : le cas de l'enseignement intégré de science et technologie au collège. Spirale, 52, 9-33.

Coquidé, M. (2015). «Se sentir vivant »: quels enjeux d'éducation biologique ? Dans M. Dell'Angelo, M.-C. Bernard, S. de Montgolfier et C. Simard (dir.), La "vie » et le "vivant": de nouveaux défis à relever dans l'éducation. SHS Web of Conferences 21. Repéré à : [http://www.shs-conferences.org/fr/articles/shsconf/ pdf/2015/08/shsconf_vv2015_03001.pdf]

Coquidé, M. (sous presse). Les rencontres de la nature à l'école. Dans C. Fleury \& A.-C. Prévot (dir.), Expériences de nature. Paris: CNRS éditions.

Dell'Angelo, M. (2007). De l'école au collège, le rapport au vivant d'élèves de 10-12 ans. Thèse de doctorat, ENS de Cachan.

Develay, M. (1997). Origines, malentendus et spécificités de la didactique. Revue Française de Pédagogie, 120, 59-66.

Forquin, J.-C. (2008). Sociologie du curriculum. Rennes : Presses universitaires de Rennes.

Hall, E. T. (1984). La danse de la vie. Temps culturel, temps vécu (traduction). Paris : Seuil.

Harlé, I., \& Lanéelle, X. (2011). Expérimentations d'enseignements scientifiques et techniques : éclairages sociologiques. Dans J. Lebeaume, A. Hasni, et I. Harlé (dir.), Recherches et expertises pour l'enseignement de la technologie, des sciences et des mathématiques (p. 41-51). Bruxelles : De Boeck.
Katz, J. (1999). An episode of whining, chapter 5. How Emotions Work (p. 41-51). Chicago: The University of Chicago Press.

Lebaume, J., \& Coquidé, M. (2002). HétérogénéitéDifférenciation : Recherches et Questions. Aster, 35, 3-16.

Lebaume, J. (2008). L'enseignement des sciences à l'école. Des leçons de choses à la technologie. Paris : Delagrave.

Lebaume, J., \& Chartier, A.-M. (dir.) (2007). La question des contenus dans la formation des maîtres. Recherche et Formation, 55.

Lebaume, J. (2011). Léducation technologique au collège: un enseignement pour questionner la refondation $\mathrm{du}$ curriculum et les réorientations des disciplines. Éducation et didactique, 5(2), 7-22.

Losego, P. (2014). Rapprocher la sociologie et les didactiques. Revue Française de Pédagogie, 188, 5-12.

Marlot, C., \& Toullec-Théry, M. (2014). Normes professionnelles et épistémologie pratique de l'enseignant: un point de vue didactique. Revue canadienne de l'éducation (RCE) / Canadian Journal of Education (CJE), 37(4). Repéré à : [http://www.cje-rce.ca/index.php/cjerce]

Marlot, C., \& Morge, L. (2015). Des normes professionnelles à caractère doxique aux difficultés de mise en œuvre de séquences d'investigation en classes de sciences : comprendre les déterminations de l'action. Recherches En Éducation, 21, 123-137. Repéré à : [http://www.recherches-en-education.net/IMG/pdf/ REE-no21.pdf]

Martinand, J.-L. (1986). Connaître et transformer la matière. Des objectifs pour l'initiation aux sciences et techniques. Berne : Peter Lang.

Martinand, J.-L. (2000). Pratiques de référence et problématique de la référence curriculaire. Dans A. Terrisse, Didactique des disciplines (p. 17-24). Bruxelles : De Boeck.

Mercier, A., Schubauer-Leoni, M., \& Sensevy, G. (2002). Vers une didactique comparée. Revue Française de Pédagogie, 141(1), 5-16.

Mujawamariya, M., Hujaleh, F., \& Lima-Kerckhoff, A. (2014). A reexamination of Ontario's science curriculum: Toward a more inclusive multicultural science education? Canadian Journal of Science, Mathematics and Technology Education, 14(3), 269-283.

Perbal, L. (2009). Laicité, matérialisme et évolution dans l'enseignement en Belgique. Dans M. Coquidé \& S. Tirard (dir.). Lévolution du vivant. Un enseignement à risque? (p. 162-163) Paris : Vuibert / Adapt.

Rayou, P. (2014). Regard complémentaire - Sociologie et didactique. Vers un espace commun de problématisation. Éducation \& Didactique, 8(1), 91-100.

Rayou, P., \& Sensevy, G. (2014). Contrat didactique et contexte sociaux. La structure d'arrière-plans des apprentissages. Revue Française de Pédagogie, 188, 23-38. 
Regnault, E. (2015). L'éducation comparée entre approche pragmatique et approche compréhensive. Mémoire d'HDR, université de Strasbourg.

Schneuwly, B. (2008). Éléments d'histoire des vingt dernières années passées et propositions conceptuelles pour la suite, in M. Brossard et J. Fijalkow (dir.), Vygotski et les recherches en éducation et en didactiques (p. 19-33). Bordeaux: Presses universitaires de Bordeaux.

Sensevy, G. (2011). Le sens du savoir. Éléments pour une théorie de l'action conjointe en didactique. Bruxelles : De Boeck.

Vidal, M. (2014). Éduquer au bien-être animal dans l'enseignement professionnel : prise en compte de l'empathie interspécifique dans le système éducatif. Thèse de doctorat, université de Toulouse.

Vinatier, I. (2009). Pour une didactique professionnelle de l'enseignement. Rennes: Presses universitaires de Rennes. 